\title{
Smart Homes or Smart Occupants? Supporting Aware Living in the Home
}

\author{
Lyn Bartram, Johnny Rodgers, and Rob Woodbury \\ School of Interactive Arts + Technology, Simon Fraser University \\ Surrey, BC, CANADA V3T 0A3 \\ $\{1 y n, j g r 3, r w\}$ asfu.ca
}

\begin{abstract}
Awareness of resource consumption in the home is a key part of reducing our ecological footprint yet lack of appropriate understanding and motivation often deters residents from behaviour change. The coming deployment of smart metering technologies, the increasing practicality of embedded devices, and the widespread use of Internet and mobile tools offer new opportunities for "greener" residents. We report on the design and implementation of a holistic interactive system that supports residents in awareness of resource use and facilitates efficient control of house systems to encourage conservation in daily activities. Initial response from two high-profile deployments in unique homes indicates this approach has great potential in engaging residents in sustainable living, but presents many challenges in how technology is integrated into the home environment.
\end{abstract}

Keywords: Residential resource use, interaction design, ubiquitous computing, information visualization, sustainability, domestic design.

\section{Introduction}

A sustainable home is more than an efficient building: it is also a living experience that encourages occupants to use fewer resources more effectively. Research has shown that small changes in behaviour, such as turning off lights, reducing heat and uncovering or covering windows, can result in energy savings between $10 \%$ and $20 \%$ [27]. Water conservation is seen as a major strategy for present and future residential needs. But changing the way we use resources is proving challenging. We envision the combination of pervasive, intelligent and interactive technologies as a powerful vehicle for reducing demand and building a conservation ethic. These technologies and patterns of use are the focus of recent research but knowledge of how they should be most effectively integrated into sustainable home design is in its infancy. We seek to enable occupants in making appropriate resource use decisions without imposing undue technological complexity or effort. In this paper we describe the design and implementation of the Aware Living Interface System (ALIS) to support and encourage sustainable living practices. We discuss the issues identified, design criteria and future work resulting from implementing the system in two unique houses. 


\subsection{A Tale of Two Houses}

Our insights and experience in building information and control systems for the aware resident arise from our involvement in the design and implementation of two sustainable homes: North House and West House.

North House is a small solar-powered home that recently placed 4th overall in the 2009 Solar Decathlon. North House incorporates sophisticated custom energy systems, adaptive intelligent building envelope technologies, specialized lighting and climate systems, and automated optimization behaviour. No conventional control systems were integrated into the home: a digital control panel and an iPhone ${ }^{\mathrm{TM}}$ provided the only means for the resident to control, track and manage energy performance in the house.

West House, our second and current project, addresses a different set of goals. It is a small, passively efficient house that uses electricity and natural gas from the public utilities and solar energy to augment heating, hot water and electricity production. It is presented as a conventional home, and typical controls (light switches, thermostats and security systems) are included throughout the house, so that digital and physical controls and feedback are intermingled. We built West House as part of our ongoing collaboration with the City of Vancouver, whose policy makers are keenly interested in how information technology, social media, alternative energies and building design can be combined to foster more sustainable living practices in "typical" houses. During the 10 days of the Solar Decathlon, North House saw more than 60,000 visitors. 65,000 people visited West House during its public display at the Vancouver 2010 Winter Olympic Games. We used these opportunities to engage in conversation and informal interviews with many different people interested in the systems.

These design cases provided us with pragmatic insights into where tradeoffs are likely to occur when deploying these systems into real-world environments. We are faced with a multitude of design constraints not typically associated with traditional approaches. The physical layout of a home introduces numerous constraints on placement, visibility, aesthetic choices, and interactive affordances. Similarly, designing for the idiosyncratic habits and expectations of users in home environments requires sensitivity to how such tools are likely to be used in everyday activities, and to how they cohere with other elements in the home. For example, residents have competing ideas about where visible technology should be located and who controls it. Simply installing software on the home PC or hanging a monitor on the wall is only going to help residents make some kinds of decisions - and only as long as it integrates coherently with their daily activities.

\section{Related Work}

The general promise of the smart home - that intelligent operation could be offloaded to a computational component - has been confounded by the human factor. The single most daunting factors in smart home automation have not been technological capability but complexity and poor usability [7]. However, significant research has focused on the power of pervasive and networked computing to automate and enable supportive and adaptive services within smart homes. This work has largely been 
targeted at enabling assistive environments for in-home care such as the Aware home [13]. A recent Apple patent [8] extends home automation to support variable control of how devices are powered and provide feedback on consumption at the device level. More recently, sensing networks have been proposed to analyse and react to user behaviour in the environment to optimize power use [11] and enable load shifting. The focus in these technologies is measurement, analysis and control of power in the home, with automation as an underlying principle. In a different approach, Weiss et al. developed a web-based application for monitoring home energy use that allows the resident to monitor consumption on a smart phone and turn individual appliances on or off [25].

Psychological research [1] shows that feedback is a central aspect of motivating resource conservation in the home. Recent web-based services partnered with power utilities approach this goal. Google PowerMeter ${ }^{\mathrm{TM}}$ and Microsoft Hohm ${ }^{\mathrm{TM}}$ allow residents to monitor and analyse aspects of their energy consumption using common "energy dashboard" displays and some description of energy use impacts. In-home displays such as Blueline's Power Cost Monitor ${ }^{\mathrm{TM}}$ show total electricity consumption in terms of $\mathrm{kWh}$ hours and money spent. Point of consumption tools such as the Kill A Watt ${ }^{\mathrm{TM}}$ are dedicated energy monitoring units attached to a particular appliance or outlet that provide numerical electrical and financial expenditure. All of these tools present aggregate data related to overall use. In contrast with these traditional computing displays, recent work has brought considerable attention to the design of "ecofeedback" technologies $[9,19]$ and visualization techniques to promote resource use awareness [12]. While others have described the design of eco-feedback technology $[9,19]$ and eco-visualization at a high level [12], our work builds on design-driven exploration of working prototype solutions in this space, similar to $[10,14]$ and fits into the Persuasive Technology and Pervasive Sensing genres that diSalvo distinguishes [6]. We depart beyond much of the HCI research, however, in that we are exploring an entire system for human-home interaction, considering both feedback and control.

How these technologies may aid or hinder residents in developing more sustainable behaviours within the home is an open question. As several researchers have found, simple data feedback is not enough. Awareness does not equate to behaviour change, and a diversity of motivations exists for conservation. Key issues [4] for residents are the lack of real time information around consumption; comprehension of what the energy use units actually mean in terms of behaviour [26]; the complexity of energymanagement devices such as programmable thermostats; poor location of feedback away from locations where resource use decisions are made [26]; and the need for motivational tools such as goal-setting abilities and social networking features $[16,18]$.

Perhaps the most widespread example of a poorly implemented human-house interactive tool is the programmable thermostat. It fails for two primary reasons. First, the interface is non-standard and invariably complex across products, so only a minority of users actually configures it successfully. More important, however, is its functional design. Most peoples' lives are more complex and variable than the simple schedules the thermostat accepts. As a result, people tend to program it for the minimum case, and end up heating their homes unnecessarily in periods when they are 
absent. This example is representative of the need for a more flexible and responsive design approach.

Chetty advocates several design principles: make real-time information visible and comprehensible; design for individual and collective agency for motivation and reward; ensure technologies are attainable; and seek new ways of stimulating discussion and engagement [4]. Fitting these technologies into the home poses additional challenges. Residents have competing ideas about where visible technology should be located and who controls it. They also feel overburdened by the complexity and inflexibility of home technologies they already use [24]. This can be seriously aggravated by automation: humans have an uneasy relationship with automated control [29], as we discovered in North House.

\subsection{Lifestyle and Human Factors}

Social scientists provide some insight into what kinds of lifestyle factors contribute to residential energy use. Research indicates four variables influence behaviour: norms and beliefs; external persuasive forces (community pressure, advertising); personal knowledge and skills; and habit and routine [23]. Energy use affected by the last is driven by comfort and effort (time and actions required to execute): conservation activities depend on lowering both cost and effort [21,22]. In addition, this research pinpoints the role of knowledge; many people have incorrect estimations of how much energy is used in hot water and appliances, influencing their use. So while demographic factors such as gender and income play major roles, the more malleable psychological aspects of habit, effort and knowledge have a strong influence.

\section{Design Rationale}

These themes were repeated in our preliminary user workshops with people who described themselves as "interested" in sustainable living environments but who had no experience with them. Participants included students, professionals and blue and white-collar workers. While the motivating models differed (some were more interested in positive financial outcomes where others were more interested in their energy footprint and ecological impact), we found several common threads. The first was time: all of our "users" identified themselves as very busy people and were concerned about having to spend too much time and effort in managing the house. A related thread was place: people are very mobile, wanted appropriate information and controls accessible from wherever they were, and wanted localized and contextually appropriate access in the house itself. For example, none found the notion of a central control panel and dashboard for lights, shutters, etc. very useful, but liked the idea of information and controls in place. A third was related to complexity: whereas several indicated they would be interested in learning more about how the house actually worked, all wanted a simple interface with a low learning curve that would provide quick access to reasonable house configuration while allowing the more expert user to fine tune settings. Finally, participants really wanted to know "how they were doing" in the context of their particular goals (financial, energy use) and how this changed over time and events. 
The design dialogue in the development of efficient buildings has largely focused on smart automation of the building systems and components for optimal performance rather than on effectively supporting how people use them [15]. But the single most daunting factors in smart home automation have not been technological capability but complexity and poor usability [7]: residents are not professional facilities managers. In contrast to the automated smart home populated with intelligent devices, we focus on the aware home with support for the smart occupant, and we focus on an integrated, extensible system as opposed to a loose collection of different tools. We aim to reduce the technological and cognitive effort required to make decisions about resource use and understand its impacts. Our rough "grounding equation" can be expressed as Cost $>$ Benefit $\neq$ Change. That is, if the perceived cost (effort, time) of doing something outweighs the perceived benefits, people are unlikely to change their behaviour [22]. We propose we need to reduce the overhead of performing conservation actions and increase the motivating benefits, including non-financial incentives. Additionally, we posit that a piecemeal approach introduces complexity, and that residents will benefit from a coherent ecosystem of information feedback and control tools that are integrated into systems they already use [2]. We based our system design on the following criteria.

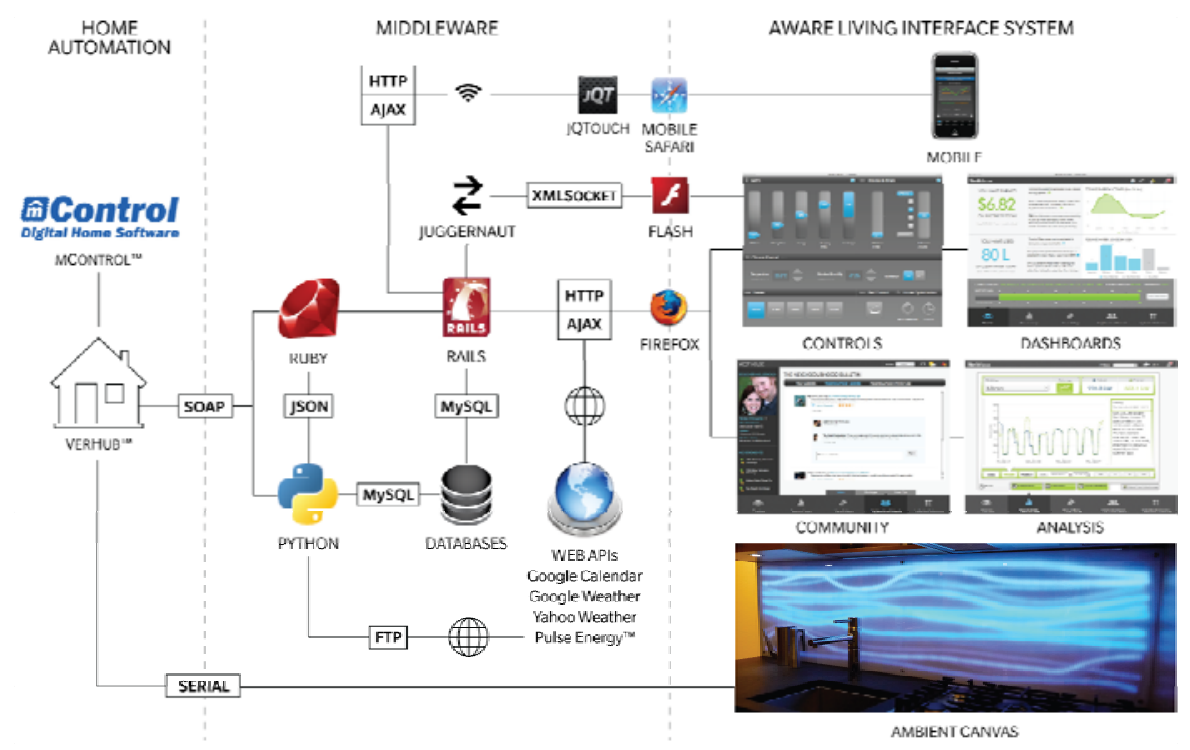

Fig. 1. The ALIS software architecture

1. Rich, real-time feedback. Make real-time and cumulative resource use information available to support decision-making and information access at a variety of levels: in-the-moment awareness; lightweight monitoring; analysis and reasoning; consequential judgment and prediction.

2. Context. Present information in contextually appropriate ways: for example, energy use expressed in both financial terms and common usage ("enough to power a washing machine"). Embed information where decisions are made. 
3. Individual and Social Motivation: Provide goal-setting capabilities and integration with social and community networks.

4. Control: Enable efficient resource use decisions by building a control hierarchy for optimizing resource conservation. Design and distribute controls appropriately: embedded in the home, remote or mobile.

5. Aesthetics: Respect the design sensibility of a home. Explore subtle ways to provide feedback.

6. Familiarity: Reduce complexity by leveraging tools people already use in their information landscape, such as calendars, browsers and clocks.

This combination of design goals is substantially more sophisticated than what available home automation systems or monitoring applications offer. In fact, it moves the design brief from that of smart home automation or energy use monitoring to a more comprehensive resource management system that is integrated with the home environment and residents' activities while respecting the dimensions of aesthetic and emotional comfort.

\section{The Aware Living Interface System}

Our system comprises three main components: a control backbone that provides finegrained measurement, device control and automation logic that is based on a

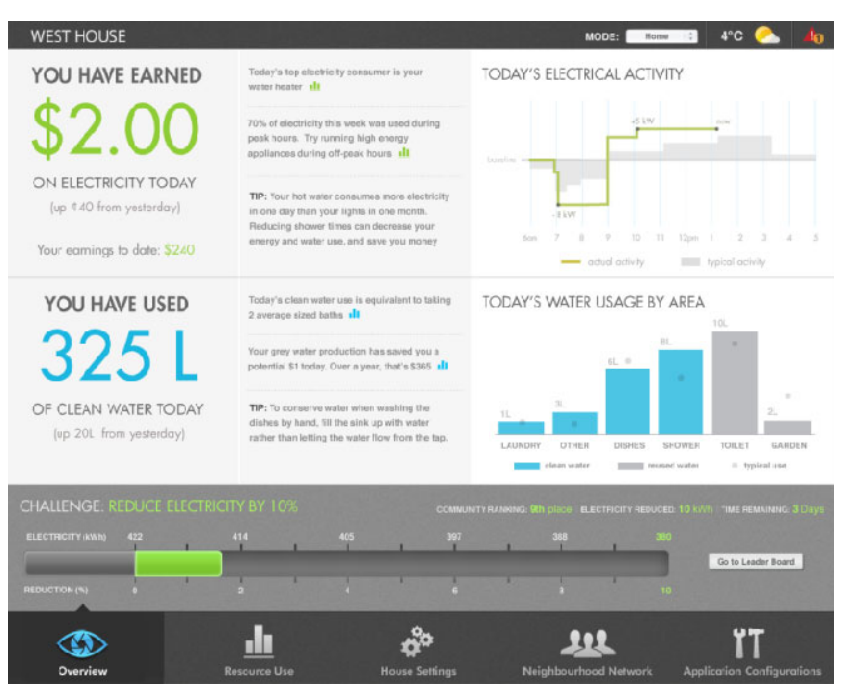

Fig. 2. The Dashboard heavily customized commercial application; a Web services layer that manages data and commands between components; and the Aware Living Interactive System (ALIS) that embodies the resident's interaction with the home (Figure 1). ALIS is built on a comprehensive information model incorporating control and device details, resourcespecific production and consumption data in terms of standard units, pricing levels, and standard usage equivalencies, personal and shared goals, and a hierarchical model of energy-control settings to enable "one-step" optimization. It currently comprises different forms: a set of variably configured client interfaces run on web browsers in several platforms; a mobile application; and ambient, "informative art" displays embedded in the home. 
ALIS supports a variety of feedback displays and analytical tools. These displays are presented as web pages running in a browser in different platforms: while they can be served to any web-enabled device, they are optimized for the viewing experience of a "typical" computer screen. We currently link to and embed information related to conservation from a number of sources, including the public utilities, directly into the ALIS information framework.

At the top level is the Resource Dashboard that expresses resource use in variable terms: as standard units, financial figures, by usage ("Today's water use is equivalent to two baths") and in relative terms ("25\% less electricity than yesterday") (Figure 2). We are exploring appropriate contextual ways to present the information, as these vary not only by individual preference, but also by location and task: for example, in the garage, the resident may wish to see the power consumed by his/her electric car overlaid on the top-level Dashboard view, and to see it in terms of "kilometers earned." Detailed information on resource production and use is available in real-time and historical views, categorized in different ways (by type of device, by location in house, by time of use). We also make use of professional building energy analysis tools from the industry leader, Pulse ${ }^{\mathrm{TM}}$ Energy, for detailed performance analysis and prediction. The application integrates visualization components in the display of energy use data in multiple formats: as numerical financial data and graphs in the Overview, comparative and scalable performance data in the Resource Usage tool, and simplified comparative performance data in the Neighbourhood Network views.

Residents can set personal milestones and challenges that can be measured by the system - for example, "use 10\% less energy than last month." Figure 2 shows the progress towards one such goal on the Dashboard.

\subsection{Social Interaction: The Neighbourhood Network}

Monthly AVG Current Month Goal

Individual challenges:

Add Change Remove

- Electricity: Reduce electricity use by $5 \%$ this month (600) in comparison to the average month (630). (hide)

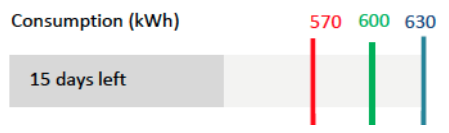

Fig. 3. Facebook App

The community interface, which we call the Neighbourhood Network, encourages competition, comparison, and collaboration between community members. It is designed to take advantage of the widespread use of social networking software to enable individuals to connect with a wider community of people pursuing similar goals around sustainability, enabling them to share strategies, incentives and successes. It is available on both PCs and mobile devices as part of the web application. Occupants can see a historical view of their energy consumption compared to a community average and set conservation goals, allowing them to compete with others in achieving those goals while sharing tips and comments. Those who follow through with their commitments receive awards (in the form of digital trophies) that are displayed to others within the community. These eco-trophies are not only applied to challenges, but also recognize the degree to which one engaged with the community by posting conservation advice and replying to other members' feedback. We note that we are not developing a social network itself; rather we integrate existing forum 
tools. A Facebook ${ }^{\mathrm{TM}}$ plug-in connects to the ALIS server to automatically collect data to publish (Figure 3): other non-ALIS data we intend to collect using voluntary self-entered reporting. Note that an issue in sharing energy use information relates to normalization: simple numbers between dissimilar houses do not support effective comparisons, so we are instead relying on the more general metric of progress towards a goal.

\subsection{Informative Art}

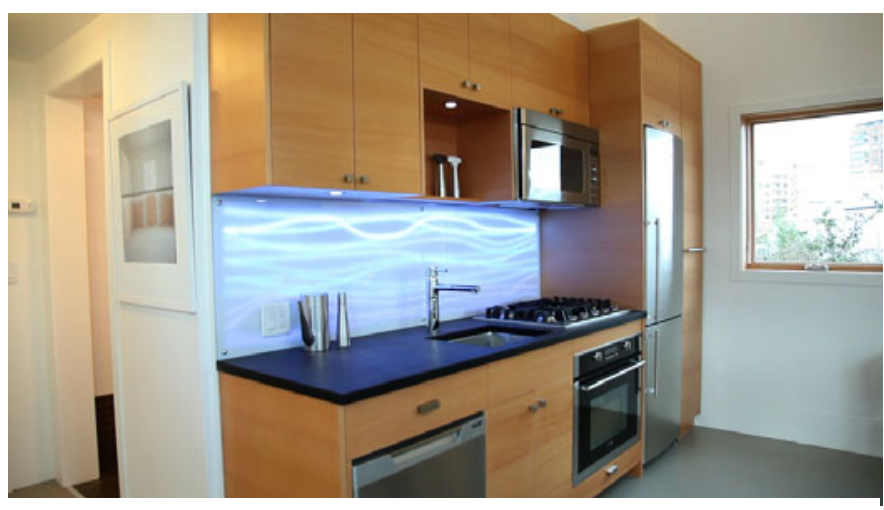

Fig. 4. The Ambient Canvas
We are exploring the use of informative art visualizations in two contexts. The Ambient Canvas is an informative art piece embedded in the kitchen backsplash that provides feedback on the use of electricity, water, and natural gas. As opposed to typical graphical displays that may use numbers or charts to convey information, the Ambient Canvas combines LED lights and filters of various materials to produce light effects on the kitchen backsplash. This subtle feedback on performance and energy efficiency does not require active attention on the part of the resident, and integrates into the home cohesively. In addition, we are currently studying the use of landscape photographs that subtly change according to levels of water consumption. These are meant to serve as both aesthetic elements and informative views. In both these contexts, informative art is intended to promote awareness of resource use to assist and influence sustainable in-themoment decisionmaking while respecting aesthetic constraints. We

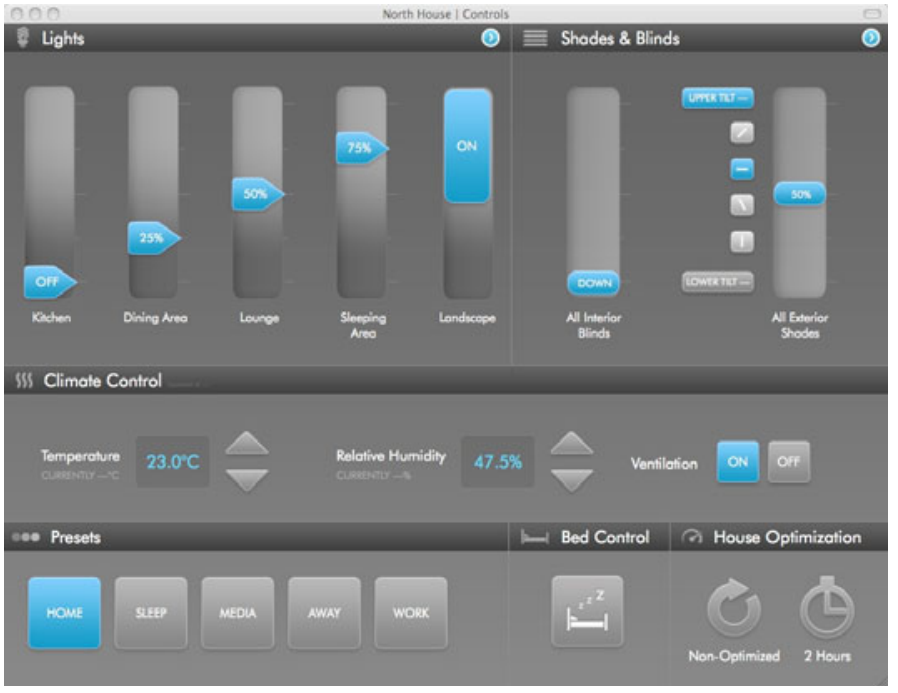

Fig. 5. One view of the ALIS controls interface 
emphasize that the attraction of such imagery is idiosyncratic to each home and are actively exploring the utility and acceptance of different representations.

\subsection{Control}

As in standard home automation, ALIS enables the resident to control and monitor lights, shades, and climate settings. In addition, the resident can configure energyoptimizing "modes" as presets in ALIS controls: for example, turning off most lights and lowering the thermostat in a "Sleep mode", or tuning settings and shutting down standby power in "Away" mode. These presets can be activated via one button from any ALIS control interface (Figure 4). Modes can also be scheduled. Modes are entirely user configurable, and coexist with individual control settings for fine-grained control when desired. Finally, we implemented a control hierarchy so that residents could easily access master controls and the most commonly used switches, or selectively drill down to fine-grained controls on demand.

\subsection{Platforms}

ALIS is currently implemented largely as a Web services application and delivered on four hardware platforms: embedded touch panel computers, personal computers, mobile phones, and the integrated informative art. The system can also accommodate expansion across further platforms. As implemented, the user interfaces provide control, feedback, and community features across the various devices, with each set of features contextualized for the location, application and form factor of the delivery hardware.

The ALIS PC interface offers the most detailed access to feedback about resource consumption in the home. It provides the resident with standard full access to the entire ALIS system. Residents use the PC interface from any computer on the Internet to configure modes, set goals, and schedule house operation.

Three touch panels are embedded into the structure of each home. Each display runs a browser in full screen kiosk mode. A large touch panel is placed in a central location: in the kitchen backsplash in North House, and on the wall in the central hallway in West House. Additionally, two smaller panels provide localized control and feedback throughout the home. In North House these are placed at the two entryways. In West House, one is installed in the garage and the other is placed beside the upstairs desk. Access to the full ALIS PC interface is possible at each access point, but complicated by the affordances and form factor of a touch screen. To address this, we have configured the interfaces on the panels to default to information and control views appropriate to their location. For example, the main control panel allows easy access

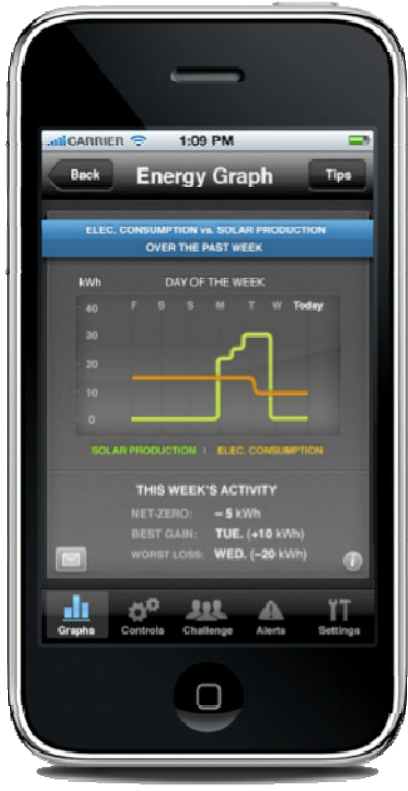

Fig. 6. Mobile 
to all house controls and the Resource Dashboard, while the garage panel provides lighting and climate controls for the garage area, one-touch home control presets, and community and transportation information. We anticipate that at this location the resident may be particularly interested in public transit schedules, carpooling options, and data on the performance of their electric car.

The mobile device provides feedback and control to the residents of the home from their pocket - a simplified remote house control. Mobile applications were developed as an extension of the web application (Figure 5). They offer a subset of the features available in the PC user interface, with each feature redesigned for use on a mobile device. For example, the controls available from the mobile emphasize ease of use through logical groupings. These "master" controls allow the resident to adjust the lights for a whole room, or shades for a whole house façade, with a single control. More fine-grained control of individual fixtures is still available, but a hierarchy of control makes the most used items easily accessible. Graphing displays are simplified; a playful 'Spinner' interface allows residents to select pairs of performance variables through a slot-machine style interaction to compare and graph on demand. The mobile application provides alerts about house status and energy consumption and production thresholds and allows residents to keep in touch with their neighbourhood network, offering challenge monitoring and community chat.

\section{Discussion}

There are many issues and challenges with meeting the design criteria we have established, and numerous open questions and investigations to pursue. Recently, West House has become tenanted as a living lab, and we will explore the system through longitudinal studies in a real-world context over the coming years. This design exercise of building the house system has given us the initial chance to assess the challenges and utility of the initial criteria.

ALIS provides a variety of views that support all levels from in-the-moment awareness to detailed analytical visualizations. These are available both via the Internet and distributed throughout the house. However, while there has been enormous interest and keen appreciation of the informative art approach, we have as yet not established what are the best mappings to communicate resource use, nor even if the simple resource use data is most appropriate to show. In North House we displayed bands of light for progress towards the resident's current goals as well as electricity production and use. In West House, the backsplash illuminates successively more as the day's usage of resources increases. We discovered immediately (and unanimously) that our visitors loved the Ambient Canvas fully lit - exactly the wrong reinforcement message to send! Similarly, we discovered in our water photos study that even some depictive images are ambiguous. While the informative art approach seems very attractive and participants and visitors very positive about it, careful attention will need to paid to the representational strategies.

We have only begun to explore contextual presentation and delivery. We do use common usage where possible to "demystify" energy use, but this is only the tip of the iceberg. Location affects meaning: water use in the laundry might be expressed in terms of loads of laundry and in the bathroom as bathtub equivalents. We have em- 
bedded information in some places where decisions are made - notably the kitchen and the living room. We are beginning to prototype small, low-data displays for appliances to simulate different time-of-day and grid/water-load conditions, anticipating the introduction of sophisticated smart metering.

ALIS' controls seamlessly integrate with the standard house devices of switches and thermostats and are ubiquitously available. Simple one-button presents allow house energy use to be finely tuned to user-configured modes, avoiding the programmable thermostat paradigm, as the resident can now simply control the house according to his or her current activity as opposed to a schedule. We are developing computational models of "effort" to evaluate how this might reduce and/or aggravate the overhead of using the house.

We have not yet fully implemented social norming tools, although currently we support a simplistic personal challenge that allows the user to set simple quantifiable goals. ALIS data can also be published to a Facebook ${ }^{\mathrm{TM}}$ plug-in that enables a community to share and compare challenges, as well as allowing the resident to compare their performance against regional performance if that data is provided by the public utilities.

Finally, we opted to build the ALIS as a web application because it allowed us to easily connect to external services and tools already in use by residents, such as Google Calendar, localized weather APIs, social networks, and energy management software tools, and to provide a familiar interface and interaction paradigm to residents through the browser. Currently we are prototyping integrating ALIS data with common information management tools such as calendars. With this, the resident can see his or her energy use in the context of daily, weekly or monthly activities. This same philosophy guided the decision to set simple one-step controls to turn the house "on" or "off"; into active or automated states. People are already used to using light switches when they leave the house or go to sleep: we simply extend the reach of that simple habit to whole-home configurations.

\section{Conclusion and Future Work}

The Aware Living Interface System is an integrated monitoring and interactive control system to encourage more efficient resource use in the home. ALIS is based on an extensible control and feedback architecture that can accommodate a diversity of energy sources, hardware devices, and home control contexts. It provides a range of user interfaces on different platforms, from smart phones and web browsers to embedded and ambient displays, each designed to support sustainable decision-making in the home. We have operationally tested the system in two very challenging public showcases, where more than 120,000 people have visited and interacted with the live systems over a combined period of 4 weeks. Our design experience with these two unique homes has helped us refine a set of criteria for building more effective information systems for residential conservation. We offer these criteria as a contribution.

Our future work will build on these lessons, and emphasize thorough evaluation of the system in a real-world context. Studying residents in this fully functional home in a vibrant neighbourhood will enable us to evaluate a variety of approaches, and 
engage with occupants and community members to further develop our understanding of social motivations. We also intend to expand the range of visualization and control devices, and to explore adaptive display and lighting control that responds to viewing requirements and resident activity. We hope this research will help to improve the design and deployment of interactive interfaces for in-home technological systems that support sustainability,

Acknowledgments. MITACS Accelerate BC, Western Economic Diversification Canada, BC Hydro, and the City of Vancouver funded this research. We sincerely thank our many industrial and government partners for their extensive support.

\section{References}

1. Abrahamse, W., Steg, L., Vlek, C., Rothengatter, T.: A review of intervention studies aimed at household energy conservation. Journal of Environmental Psychology 25(3) (2005)

2. Bartram, L., Rodgers, J., Muise, K.: Chasing the negawatt: visualization for Sustainable Living. IEEE Computer Graphics \& Applications 30(3), 8-14 (2010)

3. Chetty, M., Bernheim Brush, A.J., Meyers, B.R., Johns, P.: It's Not Easy Being Green: Understanding Home Computer Power Management. In: Proc. of the 27th Intl. Conf. on Human Factors in Computing Systems, pp. 1033-1042. ACM Press, New York (2009)

4. Chetty, M., Tran, D., Grinter, R.E.: Getting to Green: Understanding Resource Consumption in the Home. In: Proc. of the 10th Intl. Conf. on Ubiquitous Computing, pp. 242-251. ACM Press, New York (2008)

5. Clevenger, C., Haymaker, J.: The impact of the Building Occupant on Energy Simulations. In: Joint International Conference on Computing and Decision Making in Civil and Building Engineering, pp. 1-10 (2006)

6. DiSalvo, C., Sengers, P., Brynjarsdóttir, H.: Mapping the Landscape of Sustainable HCI. In: Proc. of the 28th Intl. Conf. on Human Factors in Computing Systems, pp. 1975-1984. ACM Press, New York (2010)

7. Eckl, R., MacWilliams, A.: Smart home challenges and approaches to solve them: A practical industrial perspective. In: Tavangarian, D., Kirste, T., Timmermann, D., Lucke, U., Versick, D. (eds.) IMC 2009. Communications in Computer and Information Science, vol. 53, pp. 119-130. Springer, Heidelberg (2009)

8. Fadelli, A.: Apple Inc. Intelligent Power Monitoring. U.S. Provisional Patent Application No. 61/079,751

9. Froehlich, J., Findlater, L., Landay, J.: The Design of Eco-Feedback Technology. In: Proc. of the 28th Intl. Conf. on Human Factors in Computing Systems, pp. 1999-2008. ACM Press, New York (2010)

10. Gustafsson, A., Gyllenswärd, M.: The Power-Aware Cord: Energy Awareness Through Ambient Information Display. In: Proc. of the 23rd Intl. Conf. on Human Factors in Computing Systems, Extended Abstracts, pp. 1423-1426. ACM Press, New York (2005)

11. Harle, R.K., Hopper, A.: The potential for location-aware power management. In: Proc. of UbiComp 2008, pp. 302-311 (2008)

12. Holmes, T.: Eco-Visualization: Combining Art and Technology to Reduce Energy Consumption. In: Proc. of the 6th ACM SIGCHI Conference on Creativity \& Cognition, pp. 153-162. ACM Press, New York (2007) 
13. Kientz, J.A., Patel, S.N., Jones, B., Price, E., Mynatt, E.D., Abowd, G.D.: The Georgia Tech aware home. In: CHI 2008 Extended Abstracts on Human Factors in Computing Systems, CHI 2008, Florence, Italy, April 05- 0, pp. 3675-3680. ACM, New York (2008)

14. Kim, Y., Schmid, T., Charbiwala, Z., Srivastava, M.B.: ViridiScope: Design and Implementation of a Fine-Grained Power Monitoring System for Homes. In: Proc. of the 11th Intl. Conf. on Ubiquitous Computing, pp. 245-254. ACM Press, New York (2009)

15. Leaman, A., Bordass, B.: Assessing building performance in use 4: the Probe occupant surveys and their implication. Building Research and Information 29(2), 129-143 (2001)

16. Mankoff, J., Matthews, D., Fussell, S.R., Johnson: Leveraging Social Networks to Motivate Individuals to Reduce their Ecological Footprints. In: HICSS 2007, Hawaii (2007)

17. Pierce, J., Schiano, D.J., Paulos, D.: Home, Habits, and Energy: Examining Domestic Interactions and Energy Consumption. In: Proc. of the 28th Intl. Conf. on Human Factors in Computing Systems, pp. 1985-1994. ACM Press, New York (2010)

18. Petersen, J.E., Shunturov, V., Janda, K., Platt, G., Weinberger, K.: Dormitory residents reduce electricity consumption when exposed to real-time visual feedback and incentives. International Journal of Sustainability in Higher Education 8(1), 16-33 (2007)

19. Riche, Y., Dodge, J., Metoyer, R.A.: Studying Always-On Electricity Feedback in the Home. In: Proc. of the 28th Intl. Conf. on Human Factors in Computing Systems, pp. 1995-1998. ACM Press, New York (2010)

20. Rogers, Y., Hazlewood, W.R., Marshall, P., Dalton, N., Hertrich, S.: Ambient Influence: Can Twinkly Lights Lure and Abstract Representations Trigger Behavioral Change? In: Proc. of the 12th Intl. Conf. on Ubiquitous Computing, pp. 261-270. ACM Press, New York (2010)

21. Schuitema, G., Steg, L.: Percepties van energieverbruik van huishoudelijke apparaten (Perception of energy use of domestic appliances). In: Bronner, A., Dekker, P., de Leeuw, E., de Ruyter, Smidts, A., Wieringa, J. (eds.) Ontwikkelingen in Het Marktonderzoek. Jaarboek 2005 (Developments in Marketing Research. Yearbook 2005), pp. 165-180. De Vrieseborch, Haarlem (2005)

22. Steg, L.: Promoting Household Energy Conservation. Journal of Elsevier Science, 44494453 (2008)

23. Stern, P., Dietz, T.: The value basis of environmental concern. Journal of Social Issues 50(3), 65-84 (1994)

24. Stringer, M., Fitzpatrick, G., Harris, E.: Lessons for the future: Experiences with the installation and use of today's domestic sensors and technologies. In: Fishkin, K.P., Schiele, B., Nixon, P., Quigley, A. (eds.) PERVASIVE 2006. LNCS, vol. 3968, pp. 383-399. Springer, Heidelberg (2006)

25. Weiss, M., Mattern, F., Graml, T., Staake, T., Fleisch, E.: Handy Feedback: Connecting Smart Meters with Mobile Phones. In: Proc. of the 8th Intl. Conf. on Mobile and Ubiquitous Multimedia, Article No. 15. ACM Press, New York (2009)

26. Wood, G., Newborough, M.: Energy-use information transfer for intelligent homes: Enabling energy conservation with central and local displays. Energy and Buildings 39, 495-503 (2007)

27. Woodbury, R., Bartram, L., Cole, R., Hyde, R., Macleod, D., Marques, D.: Buildings and Climate Solutions. Pacific Institute for Climate Solutions (October 2008)

28. Woodruff, A., Hasbrouck, J., Augustin, S.: A Bright Green Perspective on Sustainable Choices. In: Proc. of the 26th Intl. Conf. on Human Factors in Computing Systems, pp. 313-322. ACM Press, New York (2008)

29. Woods, D.D.: Automation: Apparent simplicity, real complexity. In: Mouloua, M., Parasuraman, R. (eds.) Human Performance in Automated Systems: Current Research and Trends, pp. 1-7. Erlbaum, Hillsdale (1994) 